\title{
WHO AML Classification of Tumors
}

National Cancer Institute

\section{Source}

National Cancer Institute. WHO AML Classification of Tumors. NCI Thesaurus. Code C159157.

A classification of acute myeloid leukemia tumors by the World Health Organization (WHO). 